\title{
Numerical Solutions of the Modified Burgers' Equation by Finite Difference Methods
}

\author{
Y. UCAR, N. M. YAGMURLU AND O. TASBOZAN
}

\begin{abstract}
In this study, a numerical solution of the modified Burgers' equation is obtained by the finite difference methods. For the solution process, two linearization techniques have been applied to get over the non-linear term existing in the equation. Then, some comparisons have been made between the obtained results and those available in the literature. Furthermore, the error norms $L_{2}$ and $L_{\infty}$ are computed and found to be sufficiently small and compatible with others in the literature. The stability analysis of the linearized finite difference equations obtained by two different linearization techniques has been separately conducted via Fourier stability analysis method.
\end{abstract}

Keywords: Modified Burgers' equation, Finite difference method, Fourier Stability Analysis.

AMS classification: 97N40, 65N30, 65D07, 76B25, 74S05,74J35

\section{INTRODUCTION}

The one-dimensional generalized Burgers' equation is of the form

$$
U_{t}+U^{p} U_{x}-v U_{x x}=0, \quad a \leq x \leq b, t \geqslant 0
$$

where $U(x, t)$ is the velocity for space $x$ and time $t, v$ is a positive constant representing thekinematic viscosity of the fluid, and $p$ is a positive parameter. When $p=1$ we get Burgers' equation, $p=2$ we get modified Burgers' equation.

The Burgers' equation has a wide range of applications in miscallenous fields as a mathematical model for several phenomena and is thus of a great interest. The analytical and numerical solutions of the equation have been found out by several authors using various methods and techniques. In the present work, a variation of it has been considered, namely the modifed Burgers' equation, given in the form of

$$
U_{t}+U^{2} U_{x}-v U_{x x}=0, \quad a \leq x \leq b
$$

where $U$ is the dependent variable, $v$ is the viscosity parameter, and $t$ and $x$ are the independent parameters, denoting time and space, recpectively. For the solution of the 
numerical example, the following boundary conditions are going to be used

$$
U(a, t)=\beta_{1}, \quad U(b, t)=\beta_{2} t \geq t_{0}
$$

The current work's main aim is to apply the finite difference methods to develop a numerical method for the approximate solution of the modified Burgers' equation. Eq. (1) has been solved both analytically and numerically by several authors using various methods and techniques. Some of them can be given as follows. The modified Burgers' equation hase been solved by Ramadan and El-Danaf [2] using the collocation method with quintic splines. The equation has been numerically solved by Ramadan et al. [3] using the collocation method with septic splines. The Burgers' and modified Burgers' equations have been solved by Saka and Dag [4] by applying time and space splitting techniques and then employed the quintic B-spline collocation procedure to approximate the resulting systems. Irk [5] has employed Crank-Nicolson central differencing scheme for the time integration and sextic B-spline functions for the space integration to the modified and time splitted modified Burgers' equation. A numerical solution has been propesed by Temsah [6] for the convection-diffusion equation using El-Gendi method with interface points and then numerical results for Burgers' and modified Burgers' equations have been shown. Grienwank and El-Danaf [7] have proposed a non-polynomial spline based method to obtain numerical solutions of the non-linear modified Burgers' equation. Bratsos [8] has used a finite-difference scheme based on rational approximations to the matrix-exponential term in a two-time level recurrence relation for the numerical solution of the modified Burgers' equation. Bratsos [9] has presented a finite-difference scheme based on fourth-order rational approximants to the matrix-exponential term in a two-time level recurrence relation for the numerical solution of the modified Burgers equation. Bratsos and Petrakis [10] have used an explicit finite difference scheme based on second-order rational approximations to the matrix-exponential term for the numerical solution of the modified Burgers' equation. The equation has been numerically solved by Roshan and Bhamra [11] by the Petrov-Galerkin method using a linear hat function as the trial function and a cubic B-spline function as the test function.

In this study, two linearization techniques have been applied to deal with the non linear term while obtaining the numerical solution of the Modified Burgers' equation. A numerical example has been considered to test the performance of two 
linearization techniques and then the stability analysis of the numerical schemes has been investigated separately.

\section{THE FINITE DIFFERENCE METHOD}

Let's suppose that the solution domain of the problem $a \leq x \leq b$ is divided into intervals having equal length $h$ in the $x$ direction and having equal time intervals $k$ in time $t$ such that $x_{i}=i h, i=0(1) N$ and $t_{j}=j k, j=0(1) J$ and $U_{i j}$ will denote $U\left(x_{i}, t_{j}\right)$ throughout the article.

In the finite difference method, in place of the dependent variable and its derivatives their approximated values by the finite difference approximation are written. These approximations will result in either a single explicit equation or a system of difference equations. When applied to non-linear problems, it normally results in non-linear system of equations and they cannot be solved directly. Thus, an appropriate numerical algorithm is used to solve them.

\section{LINEARIZATION I:}

Using the forward difference approximation for $U_{t}$, the weighted central difference approximation for $U_{x x}$ in Eq. (1) at the nodal point $(i, j+1)$

$$
U_{t} \simeq \frac{U_{i, j+1}-U_{i, j}}{k}
$$

and

$$
U_{x x} \simeq \frac{1}{h^{2}}\left(\theta\left(U_{i+1, j+1}-2 U_{i, j+1}+U_{i-1, j+1}\right)+(1-\theta)\left(U_{i+1, j}-2 U_{i, j}+U_{i-1, j}\right)\right),
$$

respectively, and applying the the following linearization technique for the nonlinear term $U^{2} U_{x}$

$$
\begin{aligned}
U^{2} U_{x} \simeq & U_{i, j+1} U_{i, j}\left(\frac{U_{i+1, j}-U_{i-1, j}}{2 h}\right)+U_{i, j} U_{i, j+1}\left(\frac{U_{i+1, j}-U_{i-1, j}}{2 h}\right)+ \\
& U_{i, j} U_{i, j}\left(\frac{U_{i+1, j+1}-U_{i-1, j+1}}{2 h}\right)-2 U_{i, j} U_{i, j}\left(\frac{U_{i+1, j}-U_{i-1, j}}{2 h}\right),
\end{aligned}
$$


we can easily obtain the following system of algebraic equations

$$
\begin{gathered}
\frac{U_{i, j+1}-U_{i, j}}{k}+U_{i, j+1} U_{i, j}\left(\frac{U_{i+1, j}-U_{i-1, j}}{2 h}\right)+U_{i, j} U_{i, j+1}\left(\frac{U_{i+1, j}-U_{i-1, j}}{2 h}\right)+ \\
U_{i, j} U_{i, j}\left(\frac{U_{i+1, j+1}-U_{i-1, j+1}}{2 h}\right)-2 U_{i, j} U_{i, j}\left(\frac{U_{i+1, j}-U_{i-1, j}}{2 h}\right)- \\
\frac{v}{h^{2}}\left(\theta\left(U_{i+1, j+1}-2 U_{i, j+1}+U_{i-1, j+1}\right)+(1-\theta)\left(U_{i+1, j}-2 U_{i, j}+U_{i-1, j}\right)\right)=0, \\
\text { for } i=1(1) N-1 \text { and } j=0(1) J .
\end{gathered}
$$

For different values of $\theta(\theta=0,1 / 2,1)$, the Eq. (3) is going to be solved using an appropriate algorithm.

\subsection{Stability analysis}

To investigate the stability of the approximation obtained by the present algorithm,we will use the von Neumann theory in which the growth factor of a typical Fourier mode is defined as:

$$
U_{m}^{n}=e^{i \beta p h} \xi^{q},
$$

where $i=\sqrt{-1}$. To investigate the stability of the numerical scheme, the nonlinear term $U^{2} U_{x}$ in the modified Burgers'equation has been linearized by making the quantity $U^{2}$ a local constant. Thus the nonlinear term in the equation converts into $\widehat{U} U_{x}$ and the Eq. (1) becomes

$$
U_{t}+\widehat{U} U_{x}-v U_{x x}=0
$$

If we take the weighted average approximation as

$$
\begin{aligned}
& \frac{U_{m, n+1}-U_{m, n}}{k}+\widehat{U}\left(\theta\left(\frac{U_{m+1, n+1}-U_{m-1, n+1}}{2 h}\right)+\right. \\
& \left.+(1-\theta)\left(\frac{U_{m+1, n}-U_{m-1, n}}{2 h}\right)\right)-\frac{v}{h^{2}}\left(\theta\left(U_{m+1, n+1}-2 U_{m, n+1}+U_{m-1, n+1}\right)+\right. \\
& \left.+(1-\theta)\left(U_{m+1, n}-2 U_{m, n}+U_{m-1, n}\right)\right)=0,
\end{aligned}
$$

the generalized $m^{\text {th }}$ row of Eq. (5) becomes

$$
\begin{aligned}
& U_{m-1}^{n+1}\left(-\frac{\theta \widehat{U}}{2 h}-\frac{v \theta}{h^{2}}\right)+U_{m}^{n+1}\left(\frac{1}{k}+\frac{2 v \theta}{h^{2}}\right)+U_{m+1}^{n+1}\left(\frac{\theta \widehat{U}}{2 h}-\frac{v \theta}{h^{2}}\right) \\
= & U_{m-1}^{n}\left(\frac{(1-\theta) \widehat{U}}{2 h}+\frac{v(1-\theta)}{h^{2}}\right)+U_{m}^{n}\left(\frac{1}{k}-\frac{2 v(1-\theta)}{h^{2}}\right)+ \\
& +U_{m+1}^{n}\left(-\frac{(1-\theta) \widehat{U}}{2 h}+\frac{v(1-\theta)}{h^{2}}\right) .
\end{aligned}
$$

Substituting the Fourier mode (4) into the linearised recurrence relationship 
yields

$$
g=\frac{a-i b}{c-i d}
$$

where

$$
\begin{aligned}
& a=h^{2}-2 k v+2 k v \theta-2 k v(\theta-1) \cos \phi, \\
& b=-k h \widehat{U}(\theta-1) \sin \phi, \\
& c=h^{2}+2 k v \theta-2 k v \theta \cos \phi, \\
& d=-k h \widehat{U} \theta \sin \phi .
\end{aligned}
$$

If $\theta=0$ is taken, it corresponds to explicit method, and the following inequality is requried for the stability condition.

$$
h^{4}-\left(h^{2}-2 k v+2 k v \cos \phi\right)^{2}-h^{2} k \widehat{U}^{2} \sin ^{2} \phi \geq 0
$$

If $\theta=1$ is taken, it corresponds to implicit method, and the following inequality is required for the system to be stable.

$$
4 h^{2} k v+4 k^{2} v^{2}-4 h^{2} k v \cos \phi-8 k^{2} v^{2} \cos \phi+4 k^{2} v^{2} \cos ^{2} \phi+h^{2} k^{2} \widehat{U}^{2} \sin ^{2} \phi \geq 0
$$

If $\theta=\frac{1}{2}$ is taken, it corresponds to Crank-Nicolson method, and the scheme is unconditionally stable by the following inequality

$$
-4 h^{2} k v(\cos \phi-1) \geq 0
$$

After some basic arithmetic operations, it is seen that the stability condition $|g| \leq 1$ is satisfied by the following inequality:

$$
c^{2}+d^{2}-a^{2}-b^{2}=96 h^{2} v \Delta t(2+\cos \phi) \sin \left[\frac{\phi}{2}\right]^{2} \geq 0
$$

therefore we have come to the conclusion that the linearised scheme is unconditionally stable. 


\section{LINEARIZATION II:}

Eq. (1) can be written as

$$
\frac{\partial U}{\partial t}+\frac{1}{p+1} \frac{\partial U^{p+1}}{\partial x}-v \frac{\partial^{2} U}{\partial x^{2}}=0 .
$$

Using the forward difference approximation for $U_{t}$, the Crank-Nicolson difference approximations for $\left(U^{p+1}\right)_{x}$ and $U_{x x}$, and then utilizing the central difference operator $\delta$ defined by $\delta_{x} U_{m, n}=U_{m+1, n}-U_{m-1, n}$ (see, e.g. [14]), Eq. (1) yields the system of algebraic equations

$$
\begin{aligned}
& \frac{U_{m, n+1}-U_{m, n}}{k}+\frac{1}{4 h(p+1)}\left\{\delta_{x}\left(U_{m, n+1}^{p+1}\right)+\delta_{x}\left(U_{m, n}^{p+1}\right)\right\}- \\
& -\frac{v}{2 h^{2}}\left(U_{m+1, n+1}-2 U_{m, n+1}+U_{m-1, n+1}+U_{m+1, n}-2 U_{m, n}+U_{m-1, n}\right)=0 .
\end{aligned}
$$

for $m=1(1) M-1$ and $n=0(1) N$.

\subsection{Stability analysis}

To investigate the stability of the above scheme, we perform the computation of Eq. (9) with the values $U_{m, n}^{*}$ instead of $U_{m, n}$. Introducing an error $E_{m, n}$ given by $E_{m, n}=U_{m, n}^{*}-U_{m, n}$ and substituting it into Eq. (9) leads to

$$
\begin{aligned}
& U_{m, n+1}^{*}-U_{m, n+1}-\left(U_{m, n}^{*}-U_{m, n}\right)+\frac{k}{(p+1) 4 h}\left\{U_{m+1, n+1}^{*^{p+1}}-U_{m+1, n+1}^{p+1}-\right. \\
& \left.\quad-\left(U_{m-1, n+1}^{* p+1}-U_{m-1, n+1}^{p+1}\right)+U_{m+1, n}^{*^{p+1}}-U_{m+1, n}^{p+1}-\left(U_{m-1, n}^{*^{p+1}}-U_{m-1, n}^{p+1}\right)\right\}- \\
& \quad-\frac{v k}{2 h^{2}}\left\{U_{m+1, n+1}^{*}-U_{m+1, n+1}-2\left(U_{m, n+1}^{*}-U_{m, n+1}\right)+\right. \\
& \left.\left.\quad+U_{m-1, n+1}^{*}-U_{m-1, n+1}+U_{m+1, n}^{*}-U_{m+1, n}\right)-2\left(U_{m, n}^{*}-U_{m, n}\right)+U_{m-1, n}^{*}-U_{m-1, n}\right\}=0 .
\end{aligned}
$$

We now assume that $U$ varies little over a small region in comparison with the errors means that

$$
U_{m-1, n} \simeq U_{m, n} \simeq U_{m+1, n} \simeq U_{m-1, n+1} \simeq U_{m, n+1} \simeq U_{m+1, n+1}
$$

It is also assumed that $E_{m, n}$ is sufficiently small compared with $U_{m, n}$ and then

$$
U_{m, n}^{*^{p+1}}-U_{m, n}^{p+1}=\left(U_{m, n}+E_{m, n}\right)^{p+1}-U_{m, n}^{p+1} \simeq(p+1) E_{m, n} U_{m, n}^{p}
$$

for all $m$ and $n$. Using the above assumptions and substituting Fourier mode $E_{m, n}=$ 
$\xi^{n} e^{i \beta m h},(i=\sqrt{-1})$ into the scheme gives the growth factor $\xi$ of the form

$$
\xi=\frac{1-4 A \sin ^{2}\left(\frac{\beta h}{2}\right)-i 2 B \sin (\beta h)}{1+4 A \sin ^{2}\left(\frac{\beta h}{2}\right)+i 2 B \sin (\beta h)}
$$

where $A=\frac{v k}{2 h^{2}}$ and $B=\frac{k U_{m, n}^{p}}{4 h}$. Taking the modulus of (10) gives $|\xi| \leq 1$. The scheme is therefore unconditionally stable.

Clearly, the scheme (9) is a non-linear system of equations in $U_{m, n+1}$ and it needs to use an iterative technique to evaluate the solution. The main aim of this study is to solve the scheme (9) by a direct method. Using a Taylor series expansion of $U_{m, n+1}^{p+1}$ about the point $(m, n)$ we obtain

$$
\begin{aligned}
U_{m, n+1}^{p+1} & =U_{m, n}^{p+1}+k \frac{\partial U_{m, n}^{p+1}}{\partial t}+\cdots \\
& =U_{m, n}^{p+1}+k \frac{\partial U_{m, n}^{p+1}}{\partial U_{m, n}} \frac{\partial U_{m, n}}{\partial t}+\cdots
\end{aligned}
$$

Hence in terms of order $k, U_{m, n+1}^{p+1} \cong U_{m, n}^{p+1}+(p+1) U_{m, n}^{p}\left(U_{m, n+1}-U_{m, n}\right)$ and taking

$$
W_{m}=U_{m, n+1}-U_{m, n}
$$

Eq. (9), with some manipulations, leads to

$$
\begin{array}{r}
\left(\frac{1}{4 h} U_{m-1, n}^{p}+\frac{v}{2 h^{2}}\right) W_{m-1}-\left(\frac{1}{k}+\frac{v}{h^{2}}\right) W_{m}+\left(\frac{v}{h^{2}}-\frac{1}{4 h} U_{m+1, n}^{p}\right) W_{m+1}= \\
\frac{1}{2 h(p+1)}\left(U_{m+1, n}^{p+1}-U_{m-1, n}^{p+1}\right)-\frac{v}{h^{2}}\left(U_{m+1, n}-2 U_{m, n}+U_{m-1, n}\right)
\end{array}
$$

$(m=1(1) M-1)$ a system of linear equations for $W_{m}$. This approximation is second order in both space and time as regards truncation error. Obviously, the solution at the $(n+1)$ th time level is obtained from (11) as $U_{m, n+1}=U_{m, n}+W_{m}$ [14].

\section{NUMERICAL EXAMPLES AND RESULTS}

For the test problem used in the present work, numerical results of the equation have been obtained and all computations have been run on a Pentium i7 PC in the Fortran code using double precision arithmetic. To show how accurate the results, both the error norm $L_{2}$ 


$$
L_{2}=\left\|U^{\text {exact }}-U_{N}\right\|_{2}=\sqrt{h \sum_{J=0}^{N}\left|U_{j}^{\text {exact }}-\left(U_{N}\right)_{j}\right|^{2}},
$$

and the error norm $L_{\infty}$

$$
L_{\infty}=\left\|U^{\text {exact }}-U_{N}\right\|_{\infty}=\max _{j}\left|U_{j}^{\text {exact }}-\left(U_{N}\right)_{j}\right| .
$$

are going to be computed and presented.

\section{TEST PROBLEM}

The analytical solution of the modified Burgers' equation is given as

$$
U(x, t)=\frac{x / t}{1+\sqrt{t} / c_{0} \exp \left(x^{2} / 4 v t\right)}, \quad t \geq t_{0}, \quad 0 \leq x \leq 1
$$

where $c_{0}$ is a constant, $0<c_{0}<1$ and $t_{0}=1$.

For the initial condition of test problem, we will take equation (15) by evaluating it at $t=1$. For the boundary conditions, we will use $U(0, t)=U_{x}(0, t)=0$ and $U(1, t)=$ $U_{x}(1, t)=0$. Various viscosity constants $v=0.01,0.001,0.005$, space steps $h=0.005$, time steps $\Delta t=0.01$ and $c_{0}=0.5$ will be taken over the problem domain $[0,1]$ during the solution process of the problem. First of all, the program has been run until the time $t=11$ and then the error norms $L_{2}$ and $L_{\infty}$ are computed and presented in Table $I$ for different values of viscosity $v$. As it is seen from the table, both of the error norms $L_{2}$ and $L_{\infty}$ are small enough. Both of the error norms $L_{2}$ and $L_{\infty}$ have been compared with those of some other authors for various values of $h$ and $v$ in Table $I I$. It is clearly seen from the table that both of the error norms are better or as good as the others found in literature. 
Table I. Comparison of the error norms $L_{2}$ and $L_{\infty}$ with $h=0.005$ and $\Delta t=0.01$ for various values of $v$.

\begin{tabular}{|c|c|c|c|c|c|c|}
\hline & \multicolumn{2}{|c|}{$v=0.01$} & \multicolumn{2}{|c|}{$v=0.005$} & \multicolumn{2}{|c|}{$v=0.001$} \\
\hline$t$ & $L_{2} \times 10^{3}$ & $L_{\infty} \times 10^{3}$ & $L_{2} \times 10^{3}$ & $L_{\infty} \times 10^{3}$ & $L_{2} \times 10^{3}$ & $L_{\infty} \times 10^{3}$ \\
\hline 1 & 0.000000 & 0.000000 & 0.000000 & 0.000000 & 0.000000 & 0.000000 \\
\hline 2 & 0.378848 & 0.816262 & 0.225949 & 0.579150 & 0.067286 & 0.259046 \\
\hline 3 & 0.344560 & 0.709949 & 0.205429 & 0.503503 & 0.061409 & 0.225358 \\
\hline 4 & 0.317165 & 0.605190 & 0.188055 & 0.429111 & 0.056346 & 0.192213 \\
\hline 5 & 0.307896 & 0.526341 & 0.175034 & 0.372673 & 0.052504 & 0.166926 \\
\hline 6 & 0.326003 & 0.525791 & 0.164589 & 0.329766 & 0.049394 & 0.147826 \\
\hline 7 & 0.369938 & 0.755043 & 0.155888 & 0.296209 & 0.046765 & 0.132819 \\
\hline 8 & 0.427983 & 0.963399 & 0.148688 & 0.269279 & 0.044486 & 0.120756 \\
\hline 9 & 0.489147 & 1.139612 & 0.143137 & 0.247219 & 0.042478 & 0.110820 \\
\hline 10 & 0.547020 & 1.281253 & 0.139607 & 0.228838 & 0.040688 & 0.102585 \\
\hline 11 & 0.598717 & 1.390450 & 0.138473 & 0.213415 & 0.039078 & 0.095516 \\
\hline
\end{tabular}

Table II. Comparison of the error norms $L_{2}$ and $L_{\infty}$ with those in other studies in the literature at $t=2,6,10$.

\begin{tabular}{|c|c|c|c|c|c|c|}
\hline & \multicolumn{2}{|c|}{$t=2$} & \multicolumn{2}{|c|}{$t=6$} & \multicolumn{2}{|c|}{$t=10$} \\
\hline & $L_{2} \times 10^{3}$ & $L_{\infty} \times 10^{3}$ & $L_{2} \times 10^{3}$ & $L_{\infty} \times 10^{3}$ & $L_{2} \times 10^{3}$ & $L_{\infty} \times 10^{3}$ \\
\hline \multicolumn{7}{|l|}{$\mathrm{h}=0.005, \Delta t=0.01, v=0.01$} \\
\hline $\operatorname{EFD}(\Delta t=0.001)$ & 0.37869 & 0.81610 & 0.32600 & 0.52579 & 0.54702 & 1.28125 \\
\hline IFD & 0.39169 & 0.83158 & 0.32683 & 0.52579 & 0.54712 & 1.28125 \\
\hline CN-FD & 0.37986 & 0.81754 & 0.32608 & 0.52579 & 0.54704 & 1.28125 \\
\hline LFD & 0.37978 & 0.81736 & 0.32605 & 0.52579 & 0.54702 & 1.28125 \\
\hline [2] & 0.52308 & 1.21698 & 0.49023 & 0.72249 & 0.64007 & 1.28124 \\
\hline [3] & 0.79043 & 1.70309 & 0.57672 & 0.76105 & 0.80026 & 1.80329 \\
\hline [5], (SBCM1) & 0.38489 & 0.82934 & - & - & 0.54826 & 1.28127 \\
\hline [5], (SBCM2) & 0.39078 & 0.82734 & - & - & 0.54612 & 1.28127 \\
\hline $\operatorname{EFD}(\Delta t=0.001),[0,1.3]$ & 0.37869 & 0.81610 & 0.27624 & 0.46512 & 0.25308 & 0.32449 \\
\hline $\mathrm{IFD},[0,1.3]$ & 0.39169 & 0.83158 & 0.27666 & 0.46734 & 0.25391 & 0.32500 \\
\hline CN-FD, $[0,1.3]$ & 0.37986 & 0.81754 & 0.27630 & 0.46533 & 0.25397 & 0.32455 \\
\hline LFD, $[0,1.3]$ & 0.37978 & 0.81735 & 0.27627 & 0.46528 & 0.25395 & 0.32452 \\
\hline [5], (SBCM1), [0,1.3] & 0.38489 & 0.82934 & - & - & 0.25586 & 0.32723 \\
\hline$[5],(\mathrm{SBCM} 2),[0,1.3]$ & 0.39078 & 0.82734 & - & - & 0.25259 & 0.32337 \\
\hline \multicolumn{7}{|l|}{$\mathrm{h}=0.005, \Delta t=0.001, v=0.005$} \\
\hline EFD & 0.22643 & 0.57988 & 0.16460 & 0.32987 & 0.13960 & 0.22886 \\
\hline IFD & 0.22781 & 0.58193 & 0.16464 & 0.33014 & 0.13958 & 0.22891 \\
\hline $\mathrm{CN}-\mathrm{FD}$ & 0.22712 & 0.58091 & 0.16462 & 0.33000 & 0.13959 & 0.22888 \\
\hline LFD & 0.22711 & 0.58090 & 0.16462 & 0.33000 & 0.13959 & 0.22888 \\
\hline [2] & 0.25786 & 0.72264 & 0.22569 & 0.43082 & 0.18735 & 0.30006 \\
\hline [5], (SBCM1) & 0.22890 & 0.58623 & - & - & 0.14042 & 0.23019 \\
\hline [5], (SBCM2) & 0.23397 & 0.58424 & - & - & 0.13747 & 0.22626 \\
\hline \multicolumn{7}{|l|}{$\mathrm{h}=0.005, \Delta t=0.01, v=0.001$} \\
\hline EFD & 0.06695 & 0.25830 & 0.04939 & 0.14773 & 0.04070 & 0.10257 \\
\hline IFD & 0.07114 & 0.26743 & 0.04951 & 0.14895 & 0.04061 & 0.10277 \\
\hline CN-FD & 0.06900 & 0.26287 & 0.04944 & 0.14834 & 0.04065 & 0.10267 \\
\hline LFD & 0.06900 & 0.26284 & 0.04944 & 0.14833 & 0.04065 & 0.10267 \\
\hline [2] & 0.06703 & 0.27967 & 0.06046 & 0.17176 & 0.05010 & 0.12129 \\
\hline [3] & 0.18355 & 0.81862 & 0.08142 & 0.21348 & 0.05512 & 0.13943 \\
\hline [5], (SBCM1) & 0.06843 & 0.26233 & - & - & 0.04080 & 0.10295 \\
\hline [5], (SBCM2) & 0.07220 & 0.25975 & - & - & 0.03871 & 0.09882 \\
\hline \multicolumn{7}{|l|}{$\mathrm{h}=0.02, \Delta t=0.01, v=0.01$} \\
\hline EFD & 0.37559 & 0.80866 & 0.32916 & 0.52579 & 0.55844 & 1.28125 \\
\hline IFD & 0.39938 & 0.83959 & 0.33067 & 0.52579 & 0.55861 & 1.28125 \\
\hline CN-FD & 0.38724 & 0.82351 & 0.32988 & 0.52579 & 0.55852 & 1.28125 \\
\hline LFD & 0.38717 & 0.82328 & 0.32982 & 0.52579 & 0.55849 & 1.28125 \\
\hline [3] & 0.79043 & 1.70309 & 0.51672 & 0.76105 & 0.80026 & 1.80239 \\
\hline [5], (SBCM1) & 0.38474 & 0.82611 & - & - & 0.55985 & 1.28127 \\
\hline [5], (SBCM2) & 0.41321 & 0.81502 & - & - & 0.55095 & 1.28127 \\
\hline
\end{tabular}

The computed numerical results together with their errors are graphed in Figures 1-3 for various values of $v$ at different time levels. But the graphs of the errors have only been drawn at time $t=10$. It can be seen that the maximum error happens at the right-hand boundary of the solution domain for $v=0.01$. However, the errors for $v=0.005$ and $v=0.001$ have been recorded around the the points where the waves 
get their highest amplitudes.
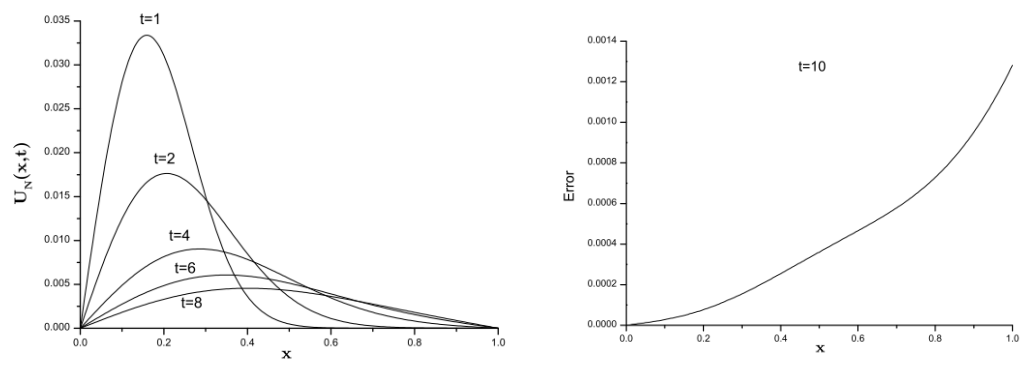

Fig. 1. The numerical solutions of Problem at different times with $v=0.01$.
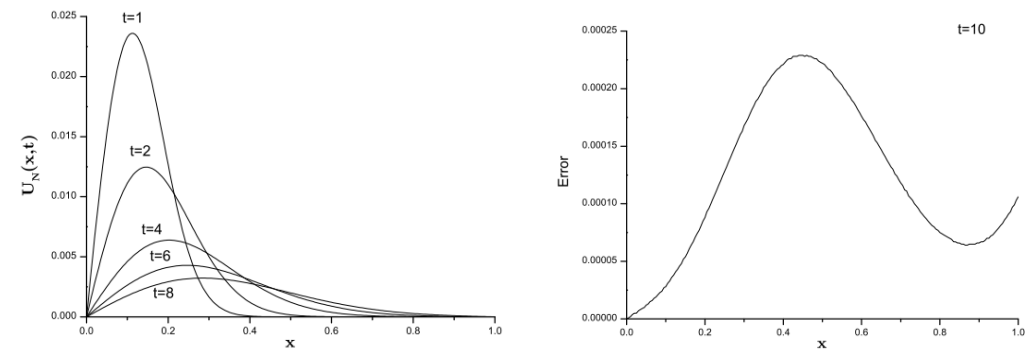

Fig. 2. The numerical solutions of Problem at different times with $v=0.005$.
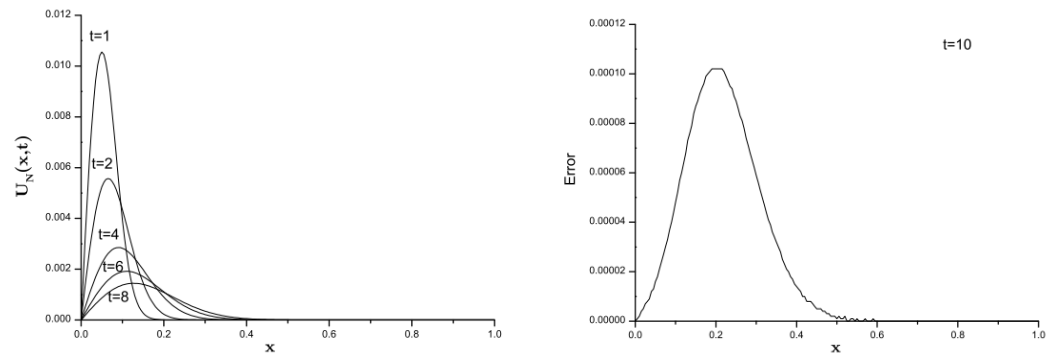

Fig. 3. The numerical solutions of Problem at different times with $v=0.001$. 


\section{CONCLUSIONS}

In the present work, numerical solutions of the Modified Burgers' equation based on the finite difference methods have been presented. To show the performance of the presented algorithm, a test problem has been considered. The performance and efficiency of the method are shown by calculating the error norms $L_{2}$ and $L_{\infty}$. The obtained results show that the error norms are sufficiently small during all computer runs. The obtained results indicate that the present method is a particularly successful numerical scheme to solve the Modified Burgers' equation. Therefore, the method used in the present work can strongly be advised to get approximate solutions of several other widely used non-linear equations in the literature.

\section{REFERENCES}

E. Hopf, The partial differential equation $U_{t}+U U_{x}-v U_{x x}=0$, Communications on Pure and Applied Mathematics, Vol. 3, pp. 201-30.

M. A. Ramadan and T. S. El-Danaf, Numerical treatment for the modified burgers equation, Matmematics and Computers in Simulation 70 (2005) 90-98.

M. A. Ramadan , T. S. El-Danaf and F. E.I. Abd Alaal, A numerical solution of the Burgers equation using septic B-splines, Chaos, Solitons and Fractals 26 (2005) 795-804.

B. Saka and I. Dag, A numerical study of the Burgers' equation, Journal of the Franklin Institute 345 (2008) 328-348.

D. Irk, Sextic B-spline collocation method for the modified Burgers' equation, Kybernetes, Vol. 38, No. 9, 2009, pp. 1599-1620.

R.S. Temsah, Numerical solutions for convection-diffusion equation using El-Gendi method, Communications in Nonlinear Science and Numerical Simulation 14 (2009) 760-769.

A. Griewank and T. S. El-Danaf, Efficient accurate numerical treatment of the modified Burgers' equation, Applicable Analysis, vol. 88, No. 1, January 2009, 75-87.

A. G. Bratsos, An implicit numerical scheme for the modified Burgers' equation, in HERCMA 2009 (9 Hellenic-European Conference on Computer Mathematics and its Applications), 24-26 September 2009, Athens, Greece.

A.G. Bratsos, A fourth-order numerical scheme for solving the modified Burgers equation, Computers and Mathematics with Applications 60 (2010) 13931400.

A. G. Bratsos and L. A. Petrakis, An explicit numerical scheme for the modified Burgers' equation, International Journal for Numerical Methods in Biomedical Engneering, 2011, 27: 232-237.

T. Roshan and K. S. Bhamra, Numerical solutions of the modified Burgers' equation by Petrov-Galerkin method, Applied Mathematics and Computation, 218 (2011) 3673-3679. 
P.M. Prenter, Splines and Variational Methods, John Wiley, New York, 1975.

S. G. Rubin and R. A. Graves, A Cubic spline approximation for problems in fluid mechanics, Nasa TR R-436, Washington, DC, 1975.

G. D. Smith, Numerical Solution of Partial Differential Equations: Finite Difference Methods, 3rd Edition, Clarendon Press, Oxford (1987).

Yusuf Ucar

Department of Mathematics, İnönü University, Malatya, 44280, Turkey

email: yusuf.ucar@inonu.edu.tr

Nuri Murat Yagmurlu

Department of Mathematics, İnönü University, Malatya, 44280, Turkey email: murat.yagmurlu@inonu.edu.tr

Orkun Tasbozan

Department of Mathematics, İnönü University, Malatya, 44280, Turkey email: orkun.tasbozan@inonu.edu.tr 